\title{
Belgeo
}

Revue belge de géographie

$3 \mid 2019$

Les petites villes européennes comme enjeu d'équité territoriale/Small European cities as stakes for territorial equity

\section{Focus sociologique sur le phénomène des espaces de coworking dans les petites et moyennes villes dans le sud-ouest de l'Allemagne}

\section{Gerhard Krauss}

\section{(2) OpenEdition}

\section{Journals}

Édition électronique

URL : http://journals.openedition.org/belgeo/34245

DOI : 10.4000/belgeo.34245

ISSN : 2294-9135

\section{Éditeur :}

National Committee of Geography of Belgium, Société Royale Belge de Géographie

\section{Référence électronique}

Gerhard Krauss, «Focus sociologique sur le phénomène des espaces de coworking dans les petites et moyennes villes dans le sud-ouest de l'Allemagne », Belgeo [En ligne], 3 | 2019, mis en ligne le 14 mai 2019, consulté le 14 février 2020. URL : http://journals.openedition.org/belgeo/34245; DOI : 10.4000/ belgeo.34245

\section{Ce document a été généré automatiquement le 14 février 2020.}

Belgeo est mis à disposition selon les termes de la licence Creative Commons Attribution 4.0 International. 


\title{
Focus sociologique sur le phénomène des espaces de coworking dans les petites et moyennes villes dans le sud-ouest de l'Allemagne
}

\author{
Gerhard Krauss
}

\section{Introduction}

1 L'objectif du présent article est d'étudier les impacts des nouvelles formes de travail collaboratif sur les territoires hors des grands centres métropolitains (périurbain, rural, petites et moyennes villes). Ces nouvelles façons de travailler sont favorisées à la fois par le développement du numérique et de l'«économie partagée " (Arvidsson, 2018), la crise de la société salariale et les tendances d'individuation dans les sociétés occidentales contemporaines touchant en particulier la population des nouveaux «travailleurs du savoir». L'originalité de l'analyse proposée ici est qu'elle prend comme base les comportements concrets des individus, leurs trajectoires sociales et professionnelles, ainsi que leur ancrage territorial.

Une vraie question est de savoir si les petites et moyennes villes pourront bénéficier à long terme des évolutions sociétales en cours dont on repère déjà les premiers signes précurseurs: une mobilité, encore marginale certes, mais croissante des travailleurs éduqués du numérique vers les petites et moyennes villes, voire les zones rurales. Il s'agit en effet d'un choix de vie murement réfléchi en toute connaissance de cause. Le désir de devenir maître de ses projets professionnels et devenir personnel l'emporte sur les inconvénients: le manque d'infrastructure en termes de services publics: transport, santé, culture, loisirs. Les conditions de travail: la mobilité subie, les 
horaires de travail contraints, doivent changer pour cette e-génération déjà autonome et nomade.

3 Certes, il s'agit ici encore d'une population assez minoritaire sur les territoires hors métropole, d'une frange spécifique des travailleurs du savoir se démarquant notamment par leur culture et éducation, ainsi que leurs parcours parfois atypiques. En effet, le contraste est grand avec les usages établis de la majorité des habitants ancrés sur ces territoires. Pour ceux-ci, l'activité professionnelle, les pratiques de mobilité et le cadre de vie continuent à être rythmés à moyen terme selon un modèle ancien développé il y a déjà plus d'un demi-siècle et qui atteint aujourd'hui ses limites, comme celles de la mobilité devenue trop coûteuse (cf. le mouvement des "gilets jaunes » sur les ronds-points).

4 Selon notre hypothèse, la population fréquentant ces espaces de coworking est susceptible de jouer un rôle pionnier dans l'avenir pour l'évolution des pratiques sur ces territoires. Le phénomène observé de la diffusion des espaces de coworking en dehors des grands centres métropolitains est révélateur d'une importance croissante de ces processus de changement. Porté donc par les travailleurs du savoir, il est nourri non seulement par les nouvelles formes de travailler liées au numérique (Gandini, 2015), mais aussi par un nouveau rapport au territoire et à la mobilité (Marzloff, 2013).

\section{Nouveaux questionnements par rapport à l'état de l'art}

5 Si différents travaux récents soulignent les effets positifs des espaces collaboratifs pour l'innovation, en étudiant les conséquences pour le management (Mérindol et al., 2016; Bauer, 2014), ou la dimension communautaire (et politique) de cette nouvelle organisation du travail (De Vaujany, 2016), ces auteurs ne s'intéressent que peu à l'ancrage territorial des nouvelles pratiques, et encore moins aux rapports changeants à la mobilité et au territoire dont témoignent les travailleurs autonomes du savoir. En outre, dans certains pays comme la France, le thème des tiers-lieux interpellant plus particulièrement les pouvoirs publics (Krauss, Tremblay, 2019), les travaux s'inscrivent ici souvent dans une démarche visant directement le lancement de politiques publiques (Lévy-Waitz, 2018). Or, les espaces de coworking, représentant une forme particulière de «tiers-lieu » (Oldenburg, 2002, 2005), ne se réduisent pas à leurs seules dimensions managériales et politiques, ou encore à leur rôle supposé central pour l'innovation. A cet égard, notre propre objectif est différent, avec comme priorité d'entamer d'abord une démarche et un temps de réflexion sur le sujet.

Une fraction grandissante des nouveaux travailleurs créatifs du savoir, en effet, montre de nouvelles exigences vis-à-vis du travail. Leurs outils de travail (ordinateur portable et accès internet) leur permettent d'échapper aux contraintes organisationnelles traditionnelles du travail et de décider plus ou moins librement où, quand et dans quelles conditions ils travaillent. De plus en plus de ces travailleurs vivent, travaillent ou souhaitent travailler et vivre en dehors des grands centres urbains. Cette tendance est bien illustrée par l'émergence de nouveaux sites internet, blogs comme «Paris je te quitte ", presse comme "Villages ", think tanks, médias dédiés etc. proposant de l'aide et des informations pratiques spécialement à ce nouveau groupe cible. Les témoignages des fondateurs de startups français selon lesquels les investisseurs américains sauraient dorénavant situer les petites villes sur la carte de France vont dans le même sens, indiquant que les endroits excentrés hébergeant des startups ou activités créatives sont 
en train d'acquérir une plus grande visibilité à l'extérieur (voir le témoignage d'Arnaud Muller, fondateur de la startup «Saagie» dans l'émission "TECH\&CO » de BFM Business du 15/11/2017). Mais beaucoup de ces travailleurs créatifs peuvent avoir des liens anciens avec ces endroits, certains retournant simplement à leur région d'origine, après avoir passé une partie de leur parcours (études, début de carrière) dans une grande ville. ${ }^{1}$

7 Ces nouvelles tendances sont susceptibles d'ouvrir de nouvelles opportunités aux petites et moyennes villes européennes.

\section{Le terrain empirique sélectionné : deux études de cas d'espaces de coworking situés dans le sud-ouest de l'Allemagne}

8 Nous proposons une étude sur le phénomène en question en nous appuyant sur les études de cas de deux espaces de coworking situés dans une commune de 8000 et une ville de 85000 habitants dans le sud-ouest de l'Allemagne. Le type d'espaces étudié est celui d'endroits monofonctionnels, focalisés uniquement sur le coworking, accueillant une population hétérogène et limitée d'usagers (une dizaine dans chaque cas), avec un modèle commercial défensif ne permettant pas de faire des bénéfices importants, correspondant plutôt à un fonctionnement « sans but lucratif » (partage des frais entre tous les utilisateurs, éventuellement avec des bénéfices très limités pour les fondateurs/animateurs du lieu, leur permettant par exemple de couvrir leurs propres loyers individuels pour l'utilisation du lieu). Nous nous intéresserons aux trajectoires sociales des utilisateurs et plus particulièrement des fondateurs de ces espaces. Enquête de terrain à l'appui, menée en décembre $2016^{2}$ dans la région du Bade-Wurtemberg, spécifique de par sa prospérité et la densité de son tissu industriel et économique. Une particularité qui sera prise en compte dans l'analyse des trajectoires des utilisateurs et fondateurs des espaces de coworking. Pourquoi ces villes moyennes et petites communes en particulier attirent-elles ces catégories de travailleurs du savoir et du numérique? Quel est leur profil et quelles sont leurs trajectoires? A première vue, il semble qu'après l'âge ultra urbain et de formation, consommateur de culture et d'infrastructure, le coworker passe sociologiquement à l'âge périurbain où l'on fonde une famille, où l'on tentera de pérenniser son activité professionnelle, sa startup (pression immobilière et désir de calme le conduisant à quitter le centre-ville).

\section{Les particularités du contexte régional}

Mais, ceci étant un schéma européen classique, contextualisons d'abord notre analyse ici en Allemagne: il s'avère que si les spécificités du territoire en question comptent aux niveaux surtout local et régional (méso), les influences du niveau sociétal (macro) ne peuvent être écartées pour autant. Ainsi, le territoire, le Bade-Wurtemberg, illustre de façon exemplaire les particularités de la société allemande : sensibilité aux problèmes environnementaux, une densité assez élevée de la population et des infrastructures, ainsi qu'un bon réseau de transports (collectif et individuel). En revanche, pour les jeunes familles dont les deux parents sont actifs, le problème de la garde des enfants (crèche, école maternelle - beaucoup plus rares en Allemagne) entre 
en jeu également. Cette trame sociétale allemande particulièrement prégnante dans ce territoire dessine d'ores et déjà un profil type du futur utilisateur d'espace de coworking qui cherche à concilier vie professionnelle, privée et mode de vie durable.

$\mathrm{Au}$ niveau de la région (méso), un certain nombre de caractéristiques économiques du territoire deviennent apparentes : le Land du Bade-Wurtemberg est l'une des régions les plus prospères d'Europe ; il représente un système d'innovation régional porté par un ensemble d'entreprises établies et des réseaux interentreprises ancrés sur le territoire (Mercedes, Porsche, Bosch, PMEs...). La culture des startups y reste cependant sous-développée (sauf dans certains secteurs technologiques très particuliers). La région se caractérise aussi par une densité démographique élevée, un tissu industriel très dense et des réseaux interentreprises bien institutionnalisés, avec, de plus, un système de transfert des technologies entre établissements de recherche et entreprises particulièrement développé, en particulier de PME. Cette région montre un faible taux de chômage ( 3 \%) et un effort de R\&D important ( 5 \% du PIB). Enfin, on constate que la nouvelle forme d'organisation du coworking semble être moins institutionnalisée dans cette région qu'ailleurs, puisque le nombre d'espaces de coworking officiellement repéré est peu élevé pour une région de cette taille et de cette densité de population (seulement $14 \mathrm{ECW}$ selon les sites officiels).

11 Les espaces de coworking n'éveillent donc qu'un intérêt limité de la part des pouvoirs publics dans cette région. Ceux-ci focalisent leurs politiques d'investissement sur les incubateurs technologiques. Les exemples étudiés représentent donc des initiatives privées n'ayant bénéficié d'aucune subvention publique.

\section{Présentation des études de cas}

12 L'un des exemples étudiés se trouve dans une ville universitaire de 85000 habitants (dont 28500 étudiants) qui s'est développée autour d'une université fondée au $15^{\mathrm{e}}$ siècle et dont les bâtiments sont éparpillés dans toute la ville, avec présence d'une grande communauté scientifique. Le deuxième exemple étudié est situé dans une commune de 8000 habitants, disposant d'une infrastructure complète (commerces, supermarchés, artisans, médecins, cabinets d'avocats, écoles...).

Les cas étudiés démontrent l'importance décisive des profils et des parcours sociaux des fondateurs pour la création et le fonctionnement de ces espaces de coworking. Les espaces étudiés n'étant pas spécialisés dans un domaine d'activité particulier, peu de collaborations professionnelles se créent entre coworkers. Celles-ci restent rares et aléatoires, favorisées par une proximité d'opportunités certes, mais non recherchées activement. Mais la communauté des coworkers à l'échelle d'un espace procure d'autres avantages. Elle est davantage tournée vers l'entre-soi que vers la quête de nouvelles collaborations ou encore le développement de réseaux socio-professionnels. Chacun est en effet spécialisé sur son propre créneau, avec un réseau professionnel spécifique et souvent déjà bien développé. De par la spécificité de la région, à l'économie stable et très implantée, les parcours des fondateurs sont sociologiquement plus interpellants. Pourquoi proposer une telle structure qui semblerait quasiment accessoire? Quant aux utilisateurs des espaces de coworking, il s'agirait simplement d'une recherche de confort, d'entre-soi. Ils seraient motivés aussi par la réussite de sortir du "home office». Dans aucun des cas étudiés, les fondateurs et utilisateurs n'ambitionnent, dans la création ou l'utilisation de leurs espaces de coworking, de 
retombées économiques pour leurs territoires. Les projets sont individuels et aléatoires.

\section{Trajectoires individuelles et attachement au lieu} les deux cas étudiés le rôle déterminant de personnalités à la fois atypiques et capables de réunir les ressources nécessaires pour faire aboutir leur projet. Celles-ci toutefois peuvent reposer sur des profils sociologiques très différents ${ }^{3}$, marqués par des parcours uniques, imprégnant ainsi le caractère spécifique de chacun de ces lieux, et par là, de manière indirecte, jetant les bases pour la pérennité ou au contraire le risque de fragilité à moyen terme des espaces de coworking ainsi créés. Enfin, à part le rôle décisif du ou des fondateurs du tiers-lieu, les utilisateurs ont un rôle à jouer également, en apportant leur propre profil qui contribue à la coloration spécifique de l'espace de coworking concerné, avec des attaches géographiques variables ${ }^{4}$.

Les comparaisons des espaces de coworking et de tiers-lieu situés dans des régions et des pays différents nous apprennent qu'il existe une très grande diversité de cas, celleci correspondant également à une diversité équivalente des trajectoires sociales et professionnelles des individus qui fondent et/ou fréquentent ces espaces (Krauss et Tremblay, 2019). En effet, la population susceptible de les fréquenter, voire de les créer, est composée en majorité d'individus extrêmement individualistes - une conséquence à la fois des évolutions contemporaines des sociétés industrielles occidentales modernes, marquées par de fortes tendances d'individuation (Beck, Beck-Gernsheim, 2009; Martuccelli, de Singly, 2012), des parcours uniques et peu standardisés, ainsi que d'un niveau de formation plutôt élevé. Et enfin, les nouveaux moyens technologiques liés au numérique facilitent également la poursuite d'objectifs personnels et individuels, tels l'attachement $\mathrm{du}$ projet professionnel au projet de vie, ou la recherche d'une compatibilité idéale entre travail (par exemple, horaires flexibles et choisis) et vie privée, des traits caractéristiques des jeunes générations des trentenaires d'aujourd'hui (voir Hurrelmann, Albrecht, 2014).

16 Ceci n'est pas contraire au principe (voire l'obligation) de coopération professionnelle, mais celle-ci exige des configurations particulières d'acteurs que les coworkers, dans nombre de cas, semblent trouver plus facilement à l'extérieur, parmi leurs connaissances professionnelles ou leurs réseaux, dans leur domaine professionnel spécifique, qu'à l'intérieur de l'espace de coworking.

17 L'attachement au lieu finalement semble pour beaucoup s'inscrire dans une perspective à durée déterminée. Toutefois, pour une majorité, l'encastrement social reste important, mais il est lié à des facteurs qui peuvent évoluer, telle la situation du conjoint, l'évolution de l'activité professionnelle, de l'entreprise, etc. Pour certains, l'ancrage local s'avère plus solide. Ils ont grandi dans la région ou parfois y ont fait leurs études, et y reviennent après des mobilités effectuées ailleurs, ou encore pour avoir un pied à terre représentant un compromis entre leur attachement social au territoire et leurs mobilités physiques et virtuelles nécessaires dans le cadre de leur activité professionnelle. Il est intéressant de noter que pour un certain nombre de coworkers, l'enracinement social sur le territoire est déterminé par la situation du conjoint : en effet, plusieurs coworkers, dans les deux espaces de coworking étudiés, ont fait part de l'importance de la situation du conjoint. Ces travailleurs autonomes du 
numérique qui peuvent facilement déménager leur activité d'une région à l'autre, voire d'un pays à l'autre, de fait suivent leur épouse ou compagne, dès lors que celle-ci est mutée ou nommée sur un poste ailleurs.

\section{Profils et parcours des personnes ayant été impliquées dans la fondation des espaces étudiés}

18 L'histoire de l'un des espaces étudiés - celui situé dans la ville universitaire de taille moyenne - commence avec un projet de construction à but social (combiné à des objectifs écologiques). Ce projet était étroitement lié au profil et parcours de son initiateur, un avocat de gauche très engagé, spécialisé en droit fiscal (choix de spécialisation peu habituel sur un territoire où la grande majorité des avocats engagés, d'orientation politique de gauche, était spécialisée en droit du travail). Grâce à son activité professionnelle, il avait une clientèle plutôt fortunée qui lui demandait régulièrement des conseils en matière de placement ou d'investissement de capital, notamment dans des domaines alternatifs et écologiques, et il réussissait donc à les convaincre d'investir leur capital disponible localement, non dans des projets écologiques, certes très populaires et nombreux sur ce territoire, mais dans l'immobilier où on pouvait aussi faire du social, en ne cherchant pas nécessairement à réaliser un bénéfice maximum.

19 Le manque de logement, en particulier pour les personnes en situation de handicap, était un grand problème sur ce territoire. Le projet, qui a fini par faire émerger le premier espace de coworking dans cette ville, était donc au départ un projet immobilier. Il visait la création d'un immeuble moderne, respectant des normes écologiques exigeantes, tout en étant exemplaire dans sa conception sociale (cohabitation de différentes populations: des appartements adaptés aux personnes handicapées en fauteuil roulant, des appartements pour des familles, des appartements en location de courte durée pour accueillir des chercheurs invités de l'université ou des établissements de recherche, des locaux pour des bureaux au rez-de-chaussée).

La ville obligeant les constructeurs à prévoir un local commercial au rez-de-chaussée (pour éviter la création de quartiers « dortoirs ", sans activité professionnelle tournée vers le quartier), cet avocat devait décider de l'utilisation du rez-de-chaussée du nouvel immeuble. Pour la décision d'y créer un espace de coworking, il s'est finalement inspiré de la lecture d'une publication spécialisée sur les bâtiments où le concept des espaces communautaires ou de coworking a été présenté.

21 Cet exemple montre le rôle central joué par des personnalités fortes et ouvertes portant des projets d'envergure qu'ils réussissent à mettre en œuvre grâce à leur travail relationnel et la mobilisation de leurs réseaux.

L'histoire de ce cas ne s'arrête cependant pas là. Le fruit de cette initiative a de fait été transmis un an après la création d'un premier espace de coworking, géré par l'initiateur lui-même, à un groupe de quatre personnes (dont deux femmes) qui refondaient à ce moment-là l'espace en le gérant entièrement à leur propre compte (il s'agissait des premiers utilisateurs, l'initiateur ne voulant plus assurer la gestion de l'espace lui-même).

23 Les profils et trajectoires de ces fondateurs sont intéressants à plusieurs égards : sur les quatre fondateurs, deux étaient des hommes ( 29 et 49 ans), dont le lien à la ville et à la 
localité, à l'origine, s'est établi en fonction de la situation et trajectoire professionnelle de leur compagne (ou épouse). Dans l'un des cas (29 ans, célibataire, en couple sans enfants), la compagne avait obtenu une place d'études à l'université, dans le deuxième (49 ans, marié, deux enfants de 22 et 15 ans), le parcours professionnel de l'épouse (médecin et chercheur en médecine) avait également déterminé la mobilité de la famille (Etats-Unis et Allemagne). Les deux personnes restantes parmi le groupe des quatre fondateurs, deux jeunes femmes (trentenaires), se distinguaient par un enracinement régional peut-être plus pérenne, marqué par des trajectoires professionnelles qui ont débuté dans la région. Tous représentaient des domaines professionnels différents, étant spécialisés dans des créneaux spécifiques.

Dans le deuxième cas d'espace de coworking étudié, situé dans une petite commune à une quinzaine de kilomètres de la prochaine ville moyenne, le fondateur (35 ans, marié, 2 enfants d'un et de trois ans) était dans une situation similaire en ce qui concerne le fait que le lien à la localité s'était au départ construit en fonction de la carrière professionnelle de son épouse. En effet, celle-ci a été nommée professeur des écoles dans un lycée de la ville moyenne voisine et le couple a décidé d'aménager dans la petite commune.

On dégage ainsi une caractéristique récurrente représentant un facteur essentiel dans un nombre important de cas: les travailleurs du numérique, surtout quand ils travaillent à leur propre compte en tant qu'indépendant, peuvent plus ou moins facilement déménager leur activité professionnelle et l'installer dans un endroit choisi, et non subi. Ainsi, ils ont les moyens d'éviter les contraintes classiques du monde du travail et adapter en grande partie leur activité professionnelle à leur projet de vie. Les espaces de coworking s'avèrent dans ce contexte être des structures utiles, permettant de faire bénéficier à chacun d'un cadre de communauté sociale, rassurant et convivial, même si ceci ne débouche pas nécessairement sur des coopérations professionnelles approfondies et durables.

\section{Trajectoires et profils des utilisateurs}

Mutations des conjoints, confort de vie et désir de rompre l'isolation du «home office ", voire d'échapper pour certains à un statut de "père au foyer ", autant de motivations pour rejoindre un espace de coworking, mais dans leurs trajectoires respectives, on retrouve aussi des situations professionnelles aux antipodes, certains très établis et à l'épreuve de tous les aléas, d'autres débutants et peu performants. S'ils maitrisent tous leurs outils et environnements numériques, ils n'ont pas tous suivi le même cursus universitaire ou professionnel. Certains y ont acquis une grande assurance, d'autres en manquent à cause d'échecs de projets, d'études, d'entreprises. Aussi si nous constatons d'abord un désir profond parmi les travailleurs autonomes $\mathrm{du}$ numérique de se regrouper, on finit par observer des communautés malgré tout hétérogènes et générant peu de coopérations professionnelles.

\section{Les communautés de coworkers}

Outre le fait, bien pensé par les fondateurs d'espaces de coworking, de faire partager les frais d'une infrastructure moderne par une communauté de coworkers liés avec le temps, par des liens qui dépassent le cadre purement professionnel, la prochaine étape 
aurait pu être la création de liens de coopérations professionnelles concrètes entre différents utilisateurs de l'espace de coworking, s'insérant dans des communautés de pratique (Cohendet et al., 2003) et favorisant ainsi l'apprentissage et l'innovation. Or, dans les deux cas d'espaces de coworking étudiés, ce phénomène est peu présent. Parmi les facteurs explicatifs, la jeunesse des deux espaces joue un rôle certainement, ainsi que leur taille (une dizaine de places dans chacun des deux espaces), et surtout l'hétérogénéité de leur composition, chacun des coworkers étant spécialisé et occupant un créneau spécifique, ce qui réduit énormément les opportunités de coopération et ainsi débouche sur peu de collaborations concrètes, à part quelques exceptions plutôt rares $^{5}$.

Si les coworkers dont la situation professionnelle paraît encore fragile ont tendance à apprécier cette hétérogénéité professionnelle qui leur permet d'éviter une situation de concurrence directe au sein d'un même espace, face à des confrères susceptibles de devenir des concurrents potentiels, les autres déjà bien établis dans leur domaine professionnel respectif ne développent pas ou peu de coopérations au sein de l'espace de coworking pour d'autres raisons : soit ils n'en voient pas la nécessité parce qu'ils disposent de leur propre réseau bien implanté (alors que le développement de nouvelles relations de coopération implique toujours des coûts de transactions plus ou moins importants), soit leurs besoins sont si spécifiques qu'ils ne correspondent pas aux prestations potentielles que peuvent offrir les autres coworkers, soit enfin, ont-ils déjà trop de relations à gérer au sein de leur propre réseau et que trop sollicités par leurs clients, ils préfèrent éviter de nouvelles coopérations.

En outre, le travail relationnel et de réseautage étant en soi une activité stratégique, le partage du réseau entre différents coworkers ne va pas de soi, et peut parfois s'avérer délicat. En effet, selon nos observations, les coworkers sont très attentifs à garder le contrôle exclusif de leur réseau professionnel, et ceci d'autant plus qu'il s'agit de relations professionnelles directement liées au cœur de métier. Pour les autres relations plus éloignées du cœur de métier, le maintien de l'exclusivité des relations est certes moins critique, mais reste néanmoins un enjeu non négligeable. La condition préalable pour pouvoir partager réellement son réseau est le principe de symétrie des échanges : faire bénéficier un coworker de son réseau nécessite une contrepartie réelle. Ainsi, un utilisateur de l'espace de coworking qui espère pouvoir bénéficier des réseaux des autres sans apporter lui-même ses propres relations susceptibles d'être utiles pour les autres risquerait bien de se faire exclure de l'accès aux réseaux par la communauté, voire d'y être marginalisé.

\section{Conclusion}

31 De l'analyse des deux espaces de coworking, nous pouvons retenir plusieurs enseignements. D'abord, il faut rappeler que les nouvelles tendances de société que l'on peut repérer dans l'émergence des espaces de coworking hors des centres métropolitains adoptent des formes variables, tout en étant marquées par des spécificités régionales. Dans le cas du sud-ouest de l'Allemagne, les espaces de coworking apparaissent à ce jour comme un phénomène accessoire, l'économie territoriale continuant à être dominée par les entreprises établies des secteurs anciens bien développés. 

technologiques de pointe, dans le soutien aux startups. Les espaces de coworking demeurant ainsi à la marge expriment ici davantage une réponse à une question sociale qu'un potentiel économique notable. Ils jouent un rôle qui ne se situe pas forcément dans la facilitation des coopérations professionnelles, des apprentissages ou des innovations. En réalité, on observe - dans les deux cas étudiés - plutôt un phénomène de repli ou d'entre-soi, ces communautés privilégiant le renforcement de liens sociaux en interne aux dépens de la construction de nouvelles relations externes. De plus, loin de déboucher sur de nouvelles coopérations professionnelles concrètes entre coworkers à l'intérieur de l'espace, les multiples interactions quotidiennes se limitent souvent à des problématiques transversales et des échanges conviviaux.

Il n'empêche que ces espaces remplissent une fonction importante pour une minorité grandissante de la population active, une frange des travailleurs qualifiés du numérique qui y trouvent une réponse ponctuelle ou pérennisable à leur quête d'une meilleure maîtrise de l'équilibrage entre vie professionnelle et privée (pour les entrepreneurs néo-ruraux, voir par exemple: Saleilles, 2006). Il s'agit pour eux de trouver un rythme de travail avec des repères et une communauté conviviale tout simplement rassurante, face aux incertitudes professionnelles auxquelles ils sont confrontés. Pour conclure, si les effets économiques immédiats et à long terme restent à prouver, on peut néanmoins constater que le recentrage d'activités dans ces espaces collaboratifs hors des métropoles contribuera peut-être à lancer de nouvelles dynamiques. Ils préfigurent en tout cas d'une nouvelle donne dans les façons de vivre et de travailler. En effet, ils expriment de par leurs implantations multi-territoriales aujourd'hui, des grands centres urbains au rural et périurbain, une volonté de changement dans la façon de travailler, de se déplacer et se retrouver.

\section{BIBLIOGRAPHIE}

ARVIDSSON A. (2018), "Value and virtue in the sharing economy", The Sociological Review Monographs, 66, 2, pp. 289-301.

BARTHE J., BESLAY C. \& GROSSETTI M. (2008), « Choix de localisation et mobilisation des ressources dans la création d'entreprises innovantes ", Géographie, économie, société, 10, 1, pp. 43-60.

BAUER W. (dir.), RIEF S., STIEFEL K.-P. \& WEISS A. (2014), The Fascination of Coworking - Potentials for Companies and their Employees, Stuttgart, Fraunhofer-Verlag.

BECK U., BECK-GERNSHEIM E. (dir.) (2009), Riskante Freiheiten : Individualisierung in modernen Gesellschaften, Suhrkamp, Frankfurt a.M.

CHAMPENOIS C. (2008), « La co-localisation d'entreprises innovantes comme non-choix. L'exemple de l'industrie allemande des biotechnologies ", Géographie, économie, société, 10, 1, pp. 61-86, doi :10.3166/ges.10.61-85.

Belgeo, 3 | 2019 
COHENDET P., CREPLET F. \& DUPOUËT O. (2003), « Innovation organisationnelle, communautés de pratique et communautés épistémiques : le cas de Linux ", Revue française de gestion, 147, nov.déc. 2003, pp. 99-121.

De VAUJANY F.-X. (coord.) (2016), Les communautés collaboratives dans la cité: De politiques pour à des politiques par les tiers-lieux ?, Rapport de recherche, Research Group on Collaborative Spaces (RGCS), <hal-01616871>.

GANDINI A. (2015), “The rise of coworking spaces : A literature review", Ephemera : theory \& politics in organization, 15, 1, pp. 193-205.

HURRELMANN K., ALBRECHT E. (2014), Die heimlichen Revolutionäre : wie die Generation Y unsere Welt verändert, Weinheim Basel, Beltz Verlag.

KRAUSS G., TREMBLAY D.-G. (dir.) (2019) (à paraître), Tiers-lieux - travailler et entreprendre sur les territoires : Espaces de coworking, fablabs, hacklabs...., Rennes, Presses Universitaires de Rennes.

LÉVY-WAITZ P. (2018), Mission coworking - territoire travail numérique : Faire ensemble pour mieux vivre ensemble, Rapport 2018, Paris, Ministère de la cohésion des territoires.

MARTIN-BRELOT H. et al. (2010), “The Spatial Mobility of the 'Creative Class': A European Perspective”, International Journal of Urban and Regional Research, 34, 4, pp. 854-870.

MARTUCCELLI D., DE SINGLY F. (2009), Les sociologies de l'individu, Paris, Armand Colin.

MARZLOFF B. (2013), Sans bureau fixe : transitions du travail, transitions des mobilités, Limoges, Fype éditions.

MÉRINDOL V., BOUQUIN N., VERSAILLES D.W., CAPDEVILA I., AUBOUIN N., LE CHAFFOTEC A., CHIOVETTA A. \& VOISIN T. (2016), Le Livre blanc des Open Labs : Quelles pratiques? Quels changements en France? Paris, Futuris/PSB newPIC.

OLDENBURG R. (2002), Celebrating the Third Place : Inspiring Stories About the "Great Good Places" at the Heart of Our Communities, Cambridge, MA, Da Capo Press.

OLDENBURG R. (2005), The Great Good Place : Cafés, Coffee Shops, Bookstores, Bars, Hair Salons, and Other Hangouts at the Heart of a Community, Philadelphia, PA, Da Capo Press.

SALEILLES S. (2006), «L'imbrication projet de vie/projet entrepreneurial chez les entrepreneurs néo-ruraux », Sciences Sociales, 1, 1, pp. 57-68.

\section{NOTES}

1. Cette observation est cohérente avec les résultats des recherches menées à l'échelle européenne concernant le rôle joué par la trajectoire personnelle (attachement géographique) dans les choix géographiques des travailleurs créatifs (Martin-Brelot et al., 2010, p. 860). Quant aux recherches sociologiques menées sur les créateurs d'entreprises et sur les colocations d'entreprises innovantes, elles ont, elles aussi, révélé l'importance de l'attachement géographique dans les projets de vie des entrepreneurs (Grossetti, 2008 ; Champenois, 2008).

2. Au total, 23 interviews semi-directives ont été réalisées dans les deux localités, dont 5 avec des fondateurs et 14 avec des utilisateurs des deux espaces, plus 4 avec d'autres informateurs.

3. Concernant les profils des fondateurs : ils étaient âgés entre 29 et 61 ans (61, 30, 29, 49, 35 ans) au moment de l'enquête avec des parcours «atypiques", souvent à la marge du marché du travail classique (avocat, designer graphique, conseiller en économie d'énergie, deux gérants d'agence internet). Tous ont fait des études (niveau IUT et/ou universitaire), mais deux parmi eux les ont arrêtées et exercent donc leur métier sans diplôme. Sur les 5, 4 étaient des hommes. 
4. Concernant les profils des utilisateurs : la moyenne d'âge se situait autour de 30 ans, en majorité des indépendants, mais il y avait aussi quelques salariés (un sixième du total), un tiers étaient des femmes; une majorité d'informaticiens-programmeurs et de gérants d'agence web, des traducteurs ( 1 indépendant et 1 salarié), quelques designers graphiques et spécialistes des médias, des ingénieurs ; la mobilité géographique de leur femme ou compagne avait déterminé le choix du lieu pour un tiers des hommes. La moitié des individus avaient des attaches préalables à la région (soit originaires de la région, soit études dans la région, soit relations professionnelles ou amicales). Parmi les coworkers, certains sont des autodidactes sans diplôme (mais minoritaires).

5. En effet, le faible niveau de coopération constaté entre la majorité des individus qui se concentrent sur leurs projets individuels semble un phénomène assez courant dans les espaces de coworking selon Arvidsson (2015, pp. 293-294). Plutôt que de représenter des communautés basées sur des coopérations professionnelles, il vaudrait mieux interpréter les espaces de coworking comme de simples « espaces de pratique », la " pratique commune » faisant office de lien entre les différents individus (Arvidsson, 2015, p. 294).

\section{RÉSUMÉS}

Les espaces de coworking se diffusent de plus en plus au-delà des centres métropolitains vers des petites et moyennes villes. L'article aborde ce phénomène sous un angle particulier, en offrant un focus sociologique sur les utilisateurs et fondateurs de ces espaces. Il s'appuie sur les études de cas de deux espaces situés dans le sud-ouest de l'Allemagne. Les espaces de coworking semblent répondre à un besoin réel d'une frange grandissante des travailleurs autonomes du numérique qui apprécient l'insertion dans une communauté humaine leur permettant d'équilibrer leur projet de vie et leur carrière professionnelle. Même si l'analyse démontre leur rôle limité pour l'accès aux réseaux, le lancement de nouvelles coopérations, ainsi que la réalisation de projets innovants, ce phénomène offre de nouvelles opportunités aux petites et moyennes villes hors des métropoles.

Coworking spaces are increasingly spreading beyond metropolitan centers to emerge in small and medium-sized cities. The article looks at this phenomenon from a particular angle, proposing a sociological focus on the users and the founders of these spaces. It is based on case studies of two spaces in south-west Germany. The coworking spaces seem to respond to a real need for a growing fringe of self-employed digital workers, who value integration in a human community, allowing them to balance their life plans and their professional careers. Although the analysis shows their limited role in accessing networks, launching new cooperations and carrying out innovative projects, this phenomenon offers new opportunities for small and medium sized towns outside the large urban centers.

\section{INDEX}

Mots-clés : Allemagne, économie créative, créatifs, représentations, petites villes Keywords : Germany, creative economy, creatives, representations, small cities 
AUTEUR

GERHARD KRAUSS

Maître de Conférences en Sociologie, Université Rennes 2, gerhard.krauss@univ-rennes2.fr 